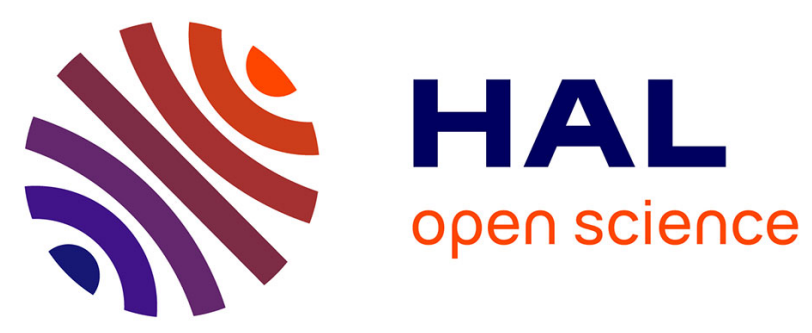

\title{
Le concept de fragilité chez la personne âgée: implications pour le rhumatologue
}

Matthieu Coulongeat, Marine Pambet, Philippe Chassagne, Jean-Bernard Gauvain

\section{- To cite this version:}

Matthieu Coulongeat, Marine Pambet, Philippe Chassagne, Jean-Bernard Gauvain. Le concept de fragilité chez la personne âgée: implications pour le rhumatologue. Revue du Rhumatisme monographies, 2019, 86, pp.164 - 171. 10.1016/j.monrhu.2019.01.005 . hal-03484731

\section{HAL Id: hal-03484731 \\ https://hal.science/hal-03484731}

Submitted on 20 Dec 2021

HAL is a multi-disciplinary open access archive for the deposit and dissemination of scientific research documents, whether they are published or not. The documents may come from teaching and research institutions in France or abroad, or from public or private research centers.
L'archive ouverte pluridisciplinaire HAL, est destinée au dépôt et à la diffusion de documents scientifiques de niveau recherche, publiés ou non, émanant des établissements d'enseignement et de recherche français ou étrangers, des laboratoires publics ou privés.

\section{(c) (1) $\$$}

Distributed under a Creative Commons Attribution - NonCommerciall 4.0 International 


\title{
Le concept de fragilité chez la personne âgée : implications pour le rhumatologue
}

\author{
Frailty concept in the elderly: practice for the rheumatologist
}

\author{
Matthieu Coulongeat ${ }^{\mathrm{a}}$, Marine Pambet ${ }^{\mathrm{a}}$, Philippe Chassagne ${ }^{\mathrm{b}}$, Jean-Bernard Gauvain ${ }^{\mathrm{a}}$ \\ a : CHR d'Orléans, Court Séjour Gériatrie, 14 Avenue de l'Hôpital, 45067 Orléans, France \\ ${ }^{\mathrm{b}}$ : CHU de Rouen, Service de médecine interne gériatrique, CHU Rouen, 76000 Rouen, \\ France
}

Correspondance : Gauvain J-B. CHR d'Orléans, Centre de Médecine Gériatrique, 14 Avenue de l'Hôpital, 45067 Orléans, jbgauvain.orleans@ gmail.com 0238744555 ou 0616338958 


\section{Résumé:}

Le syndrome de fragilité est secondaire à différents processus physiopathologiques d'intensité très variables d'un sujet à l'autre qui conduit, du fait d'une baisse des réserves d'une personne, à une décompensation fonctionnelle rapide en cas de stress telle qu'une pathologie fracturaire ou douloureuse.

La diminution des capacités de réserve va freiner les capacités à réaliser des efforts physiques chez la personne âgée. La fatigabilité et la sédentarité vont contribuer à l'ostéoporose et la sarcopénie. Laisser la fragilité s'installer contribue à ce cercle vicieux, à l'origine de conséquences comme le risque de chutes et d'événements fracturaires. La personne âgée n'est pas toujours consciente de cet état de fragilité, de son instabilité ou parfois le banalise : cet état de santé peut être vécu passivement par le sujet âgé, ses aidants ou les soignants. Or, l'enjeu est justement l'inverse : savoir repérer activement cet état Fragile dont le caractère encore réversible doit inciter à s'y intéresser.

Un événement marquant sur la santé d'un sujet âgé, comme une chute au cours de l'année passée, peut être une complication de la fragilité : elle a valeur d'alarme et témoigne d'une désadaptation de l'individu à son environnement. L'expertise rhumatologique a toute sa place chez la majorité des sujets âgés fragiles. Le repérage de facteurs de risque de fragilité a pour but de stabiliser la santé de l'individu et de contribuer à l'établissement d'une stratégie thérapeutique rhumatologique afin d'obtenir l'effet optimum que l'on attendrait chez un adulte plus jeune. Les recommandations de cette prise en charge de la fragilité participent ainsi à limiter le déconditionnement, la sarcopénie et finalement la perte d'autonomie.

Mots-clés : Fragilité, sarcopénie, comorbidités, perte d'autonomie, personnes âgées 


\section{$\underline{\text { Abstract }}$}

Multiple physiopathological processes are supported the concept of frailty syndrome. Clinical frailty presentation is heterogeneous from one subject to other mainly related to individual decrease of reserves. Frailty is associated sometimes to early functional decline in the event of stress such as fracture or painful pathology.

The decrease in reserve capacity will slow down the level of physical exercise of the elderly. Thus sedentary lifestyle will contribute to osteoporosis and sarcopenia. Let the frailty settle contribute to a vicious circle that develops is accompanied by exhaustion and insidiously increase sarcopenia, the risk of falls and fracture events. Old persons living in the community are not always aware of their frailty status and its progression, that however can be perceived by the elderly, family and relatives or caregivers. Much more, at the opposite, the challenge being to identify frailty this state whose character yet reversible should encourage interest.

A milestone event that modified the health status of an old person, such as a fall during the past year, can be a indicator of frailty. In this situation, fall must be considered as an alarm sign of a inadapted response of the old person to his surroundings. Rheumatologic skill is fully part of care for frail old people. Identifying risk factors for frailty in order to improve the health of the individual and contribute to the positive effect of treatment strategy that would be expected to be similar that in a younger adult. These recommendations to prevent Frailty may help to limit functional decline, sarcopenia and disabilities.

Keywords : Frailty, sarcopenia, comorbidity, disability, olds persons. 
Le concept de fragilité (Figure 1) [1] traduit la conséquence de l'altération de plusieurs systèmes physiologiques, de l'adaptation des capacités de réserves et de la vulnérabilité face à des changements de l'état de santé en lien avec des événements stressants. [2,3] (ex : « inflammaging », immunosénescence, insulinorésistance, fonte musculaire...). La prévalence de la fragilité dans la population Française est de $15 \%$ [4] chez les sujets de plus de 65 ans et augmente avec l'âge, elle est de l'ordre de $25 \%$ après 80 ans.

«Il n’existe pas de définition consensuelle de la fragilité. Pour la Société Française de Gériatrie et Gérontologie (SFGG) «la fragilité se définit par une diminution des capacités physiologiques de réserve qui altère les mécanismes d'adaptation au stress ». La SFGG considère que «son expression clinique est modulée par les comorbidités et des facteurs psychologiques, sociaux, économiques et comportementaux ». Le syndrome de fragilité est un marqueur de risque d'incapacités ou d'admission précoce en institution. » [1,5]. Il est associé à une surmortalité (HR : 1.63 ; IC95 \% [1.27-2.08]), à un risque d'hospitalisation accru (HR : 1.27 ; IC95 \% [1.11-1.46]) (dont les durées seront plus longues), à une perte d'indépendance fonctionnelle (HR : 1.79 ; IC95 \% [1.47-2.17]) [1]. Ces conséquences (Figure 2) [6] sont proches de celles observées au cours des états de sarcopénie [7]. Ces complications participent à la spirale du déconditionnement physique menant à la vulnérabilité (Figure 1) et secondairement à la dépendance. La fragilité est un état intermédiaire qui peut rapidement basculer vers la dépendance, cette cinétique n'est pas nécessairement irréversible si des actions, en particulier centrées sur la renutrition ou l'exercices physique, sont engagées précocement [8].

L'âge est un déterminant majeur, mais insuffisant pour expliquer à lui seul la fragilité [5]. Le phénotype initial a été décrit par L. Fried [1]. Ce modèle basé sur 5 critères principaux (Tableau 1) [1,9] aboutit à la définition de 3 profils de personnes âgées : Robuste ( 0 critère) / 
Pré-fragile ( 1 ou 2 critères) /Fragile ( $\geq 3$ critères). Au stade de pré-fragilité (en pratique stade infraclinique) les réserves physiologiques, bien que diminuées, restent suffisantes pour permettre une réponse adaptée lors d'un stress et donc une récupération complète. Le stade de fragilité est associé en revanche à une récupération plus lente ou incomplète.

\section{Les indicateurs de fragilité}

Dans sa pratique, le rhumatologue doit savoir évoquer un état de fragilité latent ou patent sur des signes généraux comme les chutes, la dénutrition («il a perdu un peu de poids »), ou même biologique lors de la mise en évidence d'une anémie chronique. Enfin et surtout, les douleurs chroniques fréquentes en rhumatologie, s'accompagnent volontiers chez le sujet âgé d'anxiété, de troubles du sommeil, d'asthénie («il sort moins fréquemment de chez lui »). Le repérage de conduite d'évitement et de réduction de la mobilité permet d'identifier ces symptômes parfois cachés ou banalisés et donc de s'interroger sur l'existence ou non d'une fragilité [10].

Le risque fracturaire (après un traumatisme de basse énergie comme une chute de sa hauteur) est corrélé non seulement à la fragilité osseuse mais aussi à d'autres caractéristiques qui sont patentes dans un contexte de fragilité. Au premier rang de ces facteurs de risque figurent le faible exercice physique et la dénutrition ayant pour conséquence la réduction associée de la masse musculaire en particulier du squelette appendiculaire [11]. Une comorbidité associée à un statut de fragilité comme une dépression ou une peur de tomber devront être simultanément appréhendées et traitées [12].

Environ $4 \%$ des patients âgés vivant à domicile et 50 à $60 \%$ des patients âgés hospitalisés sont dénutris [13]. La dénutrition est identifiée par une perte de poids de plus de $5 \%$ en 1 mois, plus de $10 \%$ en 6 mois ou un IMC $<21 \mathrm{~kg} / \mathrm{m}^{2}$. L'inflammation chronique, classique dans les maladies rhumatologiques, est un facteur de risque de dénutrition par un 
excès de catabolisme et de sarcopénie [14] souvent potentialisés par une iatrogénie médicamenteuse [15].

L'anémie (définition OMS : hémoglobine $<12 \mathrm{~g} / \mathrm{dL}$ chez la femme, et $<13 \mathrm{~g} / \mathrm{dL}$ chez l'homme) est un marqueur biologique indépendant de perte d'autonomie fonctionnelle [16] et en particulier de performance physique [17]. L'anémie n'est pas un marqueur du vieillissement normal, autrement dit n'est pas physiologique. Il est démontré que la fréquence de la fragilité était significativement supérieure chez les femmes ayant les taux d'hémoglobine les plus bas (14\%). Ainsi la probabilité d'observer une fragilité chez une femme âgée était 1,9 fois supérieure pour un taux d'hémoglobine modérément diminué (11,5 g/dL vs 13,5 g/dL) [18].

\section{Outils de repérage de la fragilité pertinents pour le rhumatologue}

A l'exemple des maladies cardio-vasculaires (ex : remplacement valvulaire aortique par voie percutanée (TAVI) ou dans le domaine de l'oncogériatrie, le bénéfice des thérapeutiques engagées (volontiers prescrites à long terme) sera différent et globalement inférieur si un état de fragilité est associé à une maladie ostéoarticulaire. Il convient donc dans des circonstances cliniques évocatrices (ex : dénutrition) de recourir à des outils permettant d'identifier ce syndrome de fragilité.

Plusieurs outils ont été proposés et discutés [19,20,21]. Un test simple, validé, de détection de la fragilité (Gerontopole Frailty Screening Tool: GFST) [22] a été créé à l'occasion d'un consensus autour des réflexions de filière gériatrique et de la démarche PAERPA (Personnes Agées En Risque de Perte d'Autonomie) [23]. Dans ce test (Figure 5), 6 items sont abordés, une dernière question prenant en compte le sentiment subjectif du médecin sur la fragilité de la personne âgée : «Votre patient vous paraît-il fragile? ». Après cette détection [24], une intervention multidimensionnelle personnalisée sera proposée (Evaluation 
Gériatrique Standardisée (EGS) ou Comprehensive Geriatric Assesment (CGA) pour les Anglosaxons). Sur 160 patients inclus dans une population communautaire âgée en moyenne de 82,7 ans, $43 \%$ avaient un risque de dénutrition et $39 \%$ un faible score d'activité physique. Si l'autonomie fonctionnelle (en l'occurrence la dépendance) pour les ADL chez ces personnes était préservée (score $=5,6 \pm 0,8 / 6$ ), les activités instrumentales de la vie courante (IADL $=6,0$ $\pm 2,3$ pour un score usuel de 8/8) étaient altérées chez 2/3 de ces patients [22].

D'autres tests très simples comme l'analyse de la vitesse de marche sur 4 mètres (Figure 3) [25] ou la mesure de la contraction isométrique au membre supérieur (dynamomètre à main) (Figure 4) permettent de repérer chez des sujets âgés autonomes et vivants à domicile un état de fragilité [26]. Ces 2 paramètres sont des facteurs prédictifs indépendants de perte d'autonomie [27]. Ils permettent également après cette phase de repérage de mesurer l'impact d'une EGS [28], le dynamomètre le permettant dès la 1re année de suivi [29]. La vitesse de marche est par ailleurs un puissant indicateur de mortalité [30], un ralentissement de 0.1 mètre/seconde étant associé à une survie diminuée de $10 \%$. Ces mesures lorsqu'elles sont significativement altérées (vitesse de marche ralentie inférieure $\leq 0.8$ mètres/secondes ou force de contraction isométrique inférieure à $20 \mathrm{~kg}$ chez la femme et $30 \mathrm{~kg}$ chez l'homme) sont de puissants indicateurs pronostics, par exemple après chirurgie orthopédique programmée ou non (ex : fracture de l'extrémité supérieure du fémur). En synthèse, s'il n'existe pas encore d'outil universel pour confirmer un état de fragilité, la mesure de la vitesse de marche peut être utilisée comme le seul outil de pratique courante d'évaluation pour son repérage [24]. Sa mesure, lorsqu'elle est associée au FRAX, permet une meilleure appréciation du risque fracturaire que l'appui monopodal [31]. En post-opératoire, de façon moins performante (faibles sensitivité et spécificité) d'une chirurgie de fracture de hanche par exemple, la mesure de la vitesse de marche permet d'apprécier le suivi et le pronostic fonctionnel [32]. Les mesures séparées de la vitesse de marche et de la force isométrique au poignet sont assez sensibles (respectivement 87,5 ; 
$100 \%)$ et spécifiques $(94,6 ; 90,5 \%)$ pour établir la présence du phénotype de fragilité de Fried, mais la combinaison de ces deux mesures s'est avérée plus sensible et spécifique que toute autre combinaison (87,5 et 99,2\%) [33]. Quant à la combinaison de la mesure préopératoire de contraction isométrique du membre supérieur couplée à la mesure de la surface du muscle psoas, elle permet de mieux apprécier l'état musculaire que chacune de ces mesures séparées et de prédire la durée de séjour [34]. Ces résultats suggèrent que pour des actes orthopédiques programmés (prothèse élective pour arthrose), ces mesures seront utiles pour repérer les sujets les plus fragiles pour leur proposer une démarche préopératoire d'activité physique adaptée/nutrition permettant d'anticiper des incapacités. Ces mesures peuvent ainsi justifier un séjour dans une unité de soins de suite et réadaptation avant l'intervention programmée.

Au total, la liste des symptômes qui conduisent au repérage de la fragilité n'est pas limitée. Les critères de Fried sont ceux qui sont les plus connus et qui ont permis dans nombre d'études de conforter le concept de fragilité. L'important pour le clinicien sera de les rattacher à une attitude d'évitement dans les activités de l'individu au moment de l'anamnèse. Confirmer par une vitesse de marche l'hypothèse d'un statut de fragilité, permettra de discuter l'intérêt d'une concertation gériatrique. Une prise en charge associée plus globale (plan personnalisé de soins après EGS) aura alors pour but d'assurer de meilleures chances de réussite thérapeutique. L'important pour le rhumatologue comme pour le gériatre est de s'attacher à repérer les éléments qui conduiraient à une perte de mobilité et un risque de perte d'indépendance. Ainsi pour atteindre l'objectif du meilleur aspect fonctionnel possible, indépendamment de la stratégie thérapeutique rhumatologique, deux questions pratiques doivent donc animer le rhumatologue soignant la personne âgée :

1- est-elle «fragile»? (réponse positive si la vitesse de marche est $\leq 0,8 \mathrm{~m} / \mathrm{sec}$ )

2- puis est-elle « sarcopénique»? 
La sarcopénie désigne la perte de masse musculaire liée à l'âge. Sa recherche en clinique repose sur la mesure de trois paramètres [35] : la performance physique (vitesse de marche $<0,8 \mathrm{~m} / \mathrm{sec}$ pour les hommes ou les femmes), la force musculaire (seuil au dynamomètre $<30 \mathrm{~kg}$ pour les hommes, < $20 \mathrm{~kg}$ pour les femmes), la masse musculaire appendiculaire (impédancemétrie ou absorptiométrie : $10,76 \mathrm{Kg} / \mathrm{m} 2$ pour les hommes, $6,75 \mathrm{Kg} / \mathrm{m} 2$ pour les femmes).

Les critères EWGSOP permettent de répondre à cette question, indépendamment de l'âge [36], par la recherche de deux critères [35] :

- Un critère de masse musculaire (par impédancemétrie, absorptiométrie biphotonique, en leur absence une surface musculaire brachiale (SMB) : $\leq 21,4 \mathrm{~cm}^{2}$ pour les Hommes, $\leq 21,6 \mathrm{~cm}^{2}$ pour les Femmes), en sachant que les évaluations de la masse musculaire par scanner lombaire (en regarde de L3) ou par absorptiométrie biphotonique restent des méthodes de référence mais sont rarement réalisées en pratique courante.

- Un critère de performance (vitesse de marche) ou de force musculaire (dynamomètre) A noter qu'en l'absence d'impédancemétrie ou d'absorptiomètre, l'anthropométrie (en faisant l'hypothèse que le bras a un pourtour circulaire et que la SMB -Surface Musculaire Brachialeest un témoin de la masse musculaire totale) permet d'apprécier la masse musculaire (calcul résumé dans le tableau 2) après avoir mesuré la circonférence brachiale et l'épaisseur du pli tricipital).

Sur un plan clinique, la majorité des patients sarcopéniques sont physiquement fragiles : la prise en charge de la sarcopénie est plus amplement développée dans un autre chapitre de cette monographie.

\section{4 - Concept de fragilité en rhumatologie : en pratique}


La prise en charge globale des sujets âgés avec pathologie rhumatologique est complexe en présence de plusieurs comorbidités et d'une polymédicamentation. Le concept de fragilité implique une prise en charge globale de la personne âgée qui sera intégrée aux choix thérapeutiques envisagés. La prise en charge de l'arthrose, des conséquences des chutes et de l'ostéoporose (prévention primaire ou secondaire) et bien entendu des rhumatismes inflammatoires chroniques constituent des exemples non exhaustifs où le rhumatologue devra appréhender simultanément l'existence d'une fragilité et ses composants.

\section{1 - Arthrose et fragilité.}

La fragilité chez des patients arthrosiques a été étudiée à travers 2 indicateurs :

- les critères de Fried [1], où dans une population de personnes âgées de 60 ans souffrant d'arthrose la prévalence des états pré-fragiles et fragiles était respectivement de 42,6 à $51 \%$ et de 10,2 à 14,1\% [37,38]. En analyse multivariée, les facteurs le plus souvent associés à la fragilité (parmi les 5 composants du phénotype de Fried) étaient la perte de poids, la notion d'épuisement, la vitesse de marche et la force de contraction isométrique [38].

- l'index de fragilité de Groninguen (GF1) [39] qui comprend 15 items explorant 8 domaines de fragilité (mobilité, condition physique, vision, audition, alimentation, multimorbidité, cognition, tissu psychosocial). Un score $\geq 4$ traduit une fragilité.

Chez les sujets fragiles ayant bénéficié d'une d'arthroplastie totale de hanche (implantation primaire ou reprise), comparativement aux sujets robustes, la mortalité mesurée à un an était 5 fois supérieure (HR : 5.65 ; IC95 \% [3.28-9.71]). La fréquence des complications post-opératoires (infections (HR : 3.63 ; IC95 \% [2.33-5.65]), hématomes (HR : 2.61 ; IC95 \% [2.02-3.36]), rupture de matériel (HR : 2.10 ; IC95 \% [1.5-2.95]) et de réinterventions (HR : 2.22 ; IC95 \% [1.9-2.59]) était significativement supérieure chez les malades fragiles [40]. 


\section{2- Rhumatismes inflammatoires}

Le modèle «treat-to-target » appliqué à la polyarthrite rhumatoïde $(\mathrm{PR})$ ne semble pas aussi facile à mettre en place chez le sujet âgé que chez l'adulte jeune [41]. Chez 124 malades, âgés en moyenne de 50 ans et suivis 2 années, l'intensité douloureuse, chez les sujets fragiles, était significativement supérieure en cas de fragilité, comparativement aux sujets robustes, et l'autonomie fonctionnelle était moins bonne àterme (RR : 0.44 ; IC95 \% [0.19$0.69])[42]$.

La présence d'un ou plusieurs paramètres tels que le nombre de comorbidités, la fragilité, le déclin cognitif et/ou physique, la dépendance et la polymédication conduisent les rhumatologues à modifier leurs pratiques, par exemple en initiant moins souvent et à plus petites doses un traitement de fond [41].

\section{3- Les chutes}

Chez les sujets fragiles :

1/ le risque de chute est supérieur à celui observé chez des personnes non fragiles (OR : 2,41; IC $95 \%$ [1,93-3,01]) et cela indépendamment de leur DMO. [43]

2/ le risque de fracture de l'extrémité supérieure du fémur est majoré (HR : 1,70 ; IC $95 \%[1,35-2,15])$ comparativement à des sujets appariés pour l'âge et non fragiles. [43]

3/ Les patients ayant plus de 3 fractures vertébrales sont plus souvent fragiles (OR : 9.2; IC95 \% [1.5-55.5]), contrairement aux patients ayant un IMC normal (OR : 0.704 ; IC95 \% [0.543-0.913])[44]. Si un antécédent de fracture reste un facteur de risque majeur à rechercher, la notion de chute prévalente est un facteur déterminant pour l'estimation du risque fracturaire de ces personnes âgées [45]. Toute notion de chute ou de fracture dans l'histoire d'un patient âgé, motivera donc la recherche d'une fragilité associée [8]. Les tests de repérage des patients âgés à risque de chute restent intéressants à pratiquer au cours d'une évaluation de risque 
fracturaire : appui monopodal, Get Up et pieds tandem étant les plus rapides et fiables en pratique clinique (Tableau 3)[46,47,48]. Les dernières recommandations du Groupe de Recherche et d'Information sur les Ostéoporoses (GRIO) portant sur le traitement antiostéoporotique soulignent l'intérêt de la prévention des chutes dans le but de réduire le risque fracturaire [49].

\section{4- Vitamine D abaissée, un marqueur de fragilité chez le sujet âgé chuteur}

L'insuffisance en $25 \mathrm{OH}$ Vitamine D $(25(\mathrm{OH}) \mathrm{D})$ (calcidiol) est un des éléments importants au carrefour d'une réflexion tridimensionnelle «ostéopathie raréfiante / chutes / fragilité » [50]. Aux effets osseux, s'ajoutent les bénéfices en termes de réduction du risque de chute et de préservation de la fonction musculaire lorsque des taux sériques de $25(\mathrm{OH}) \mathrm{D}$ sont supérieurs à $30 \mathrm{ng} / \mathrm{mL}(75 \mathrm{nmol} / \mathrm{L})$ [51].

Des taux sériques bas de 25(OH)D sont associés significativement : à une surmortalité [49] chez des résidentes d'EHPAD (HR : 1,56 ; IC95 \% [1,01-2,4]) [52] mais aussi dans une population communautaire. [51,53-56]

Dans une vision dynamique de la fragilité, la relation entre l'insuffisance en 25(OH)D et ses états transitionnels a été étudiée [57]. L'hypothèse était que les personnes avec les niveaux les plus élevés en $25(\mathrm{OH}) \mathrm{D}$ évoluaient plus lentement vers un état de fragilité. Le statut de 25(OH)D en lien avec une fragilité a été étudié chez 1155 personnes âgées de 65 ans et plus lors d'un suivi de cohorte de 6 années. Un statut de «pré-fragilité » avec un taux de 25(OH)D préservé était un élément de bon pronostic pour une récupération potentielle; les changements de statut d'état des profils « robuste » et « fragile» n'étaient pas statistiquement significatifs ; par contre, chez les «pré-fragiles », chaque diminution du taux de $5 \mathrm{ng} / \mathrm{ml}$ de 25(OH)D était plutôt associée à un risque supérieur de décès (OR : 1,46 ; IC95 \% [1,18-2,07]) et un risque de fragilité incidente de 1,13 plus importante (IC95 \% [0,9-1,39]) par rapport à ceux capables d'une réversibilité vers la robustesse. 


\section{5- Ostéoporose : sujet âgé « fragile » fracturé et valorisation du traitement}

Contrairement aux idées reçues, les bénéfices d'un traitement de fond sont supérieurs chez les sujets les plus fragiles [50]. C'est donc sur les plus fragiles que l'adhésion au traitement de fond devra être optimale. Dans une étude rétrospective observationnelle [58] de 103852 femmes âgées de 65 ans ou plus, un suivi fracturaire sur 12 mois avant et après une fracture prévalente a été réalisé. L'adhésion au traitement de fond anti-ostéoporotique (Medication Possession Ratio (MPR) : rapport du nombre de comprimés qui auraient dû être pris sur le nombre de comprimés prescrits) était moins bonne chez les sujets les plus fragiles.

Déclaration d'intérêts : Les auteurs ne déclarent aucun conflit d'intérêt avec la thématique de cet article.

Remerciements : au Dr J. Biogeau (CHR d'Orléans) pour son aide bibliographique et au Pr Y. Rolland (CHU de Toulouse) pour sa lecture méthodologique. 


\section{Références}

1. Fried LP, Tangen CM, Walston J, Newman AB, Hirsch C, Gottdiener J, et al. Frailty in older adults: evidence for a phenotype. J Gerontol A Biol Sci Med Sci. mars 2001;56(3):M146156.

2. Clegg A, Young J, Iliffe S, Rikkert MO, Rockwood K. Frailty in elderly people. Lancet. 2 mars 2013;381(9868):752-62.

3. Lang PO, Trivalle C, Dramé M. La fragilité : mythe ou réalité ? NPG Neurologie Psychiatrie - Gériatrie. août 2012;12(70):163-70.

4. Santos-Eggimann B, Cuénoud P, Spagnoli J, Junod J. Prevalence of frailty in middle-aged and older community-dwelling Europeans living in 10 countries. J Gerontol A Biol Sci Med Sci. juin 2009;64(6):675-81.

5. Rolland Y, Benetos A, Gentric A, Ankri J, Blanchard F, Bonnefoy M, et al. [Frailty in older population: a brief position paper from the French society of geriatrics and gerontology]. Geriatr Psychol Neuropsychiatr Vieil. déc 2011;9(4):387-90.

6. Cesari M, Landi F, Vellas B, Bernabei R, Marzetti E. Sarcopenia and physical frailty: two sides of the same coin. Front Aging Neurosci. 2014;6:192.

7. Michel J-P, Lang P-O. Quelles sont les relations entre sarcopénie et fragilité ? NPG Neurologie - Psychiatrie - Gériatrie. déc 2011;11(66):229-31.

8. INSERM. Expertise collective Inserm. Rapport complet. Activité physique et prévention des chutes chez les personnes âgées. Rapport. In: Expertise collective. Paris: Les éditions Inserm; 2015. p. XII-508 p. Disponible sur: http://hdl.handle.net/10608/6807

9. Radloff LS. The CES-D Scale: A Self-Report Depression Scale for Research in the General Population. Applied Psychological Measurement. juin 1977;1(3):385-401.

10. Brennan F, Carr DB, Cousins M. Pain management: a fundamental human right. Anesth Analg. juill 2007;105(1):205-21.

11. Sambrook PN, Cameron ID, Chen JS, Cumming RG, Lord SR, March LM, et al. Influence of fall related factors and bone strength on fracture risk in the frail elderly. Osteoporos Int. mai 2007;18(5):603-10.

12. Kressig RW, Wolf SL, Sattin RW, O’Grady M, Greenspan A, Curns A, et al. Associations of demographic, functional, and behavioral characteristics with activity-related fear of falling among older adults transitioning to frailty. J Am Geriatr Soc. nov 2001;49(11):1456 $-62$.

13. Ferry M, Mischlich D. Nutrition de la personne âgée: [aspects fondamentaux, cliniques et psycho-sociaux. Issy-les-Moulineaux: Elsevier-Masson; 2012.

14. Wang J, Leung K-S, Chow SK-H, Cheung W-H. Inflammation and age-associated skeletal muscle deterioration (sarcopaenia). J Orthop Translat. juill 2017;10:94-101. 
15. Morley JE. Anorexia of aging: physiologic and pathologic. Am J Clin Nutr. oct 1997;66(4):760-73.

16. Denny SD, Kuchibhatla MN, Cohen HJ. Impact of anemia on mortality, cognition, and function in community-dwelling elderly. Am J Med. avr 2006;119(4):327-34.

17. Penninx BWJH, Guralnik JM, Onder G, Ferrucci L, Wallace RB, Pahor M. Anemia and decline in physical performance among older persons. Am J Med. 1 août 2003;115(2):10410.

18. Chaves PHM, Semba RD, Leng SX, Woodman RC, Ferrucci L, Guralnik JM, et al. Impact of anemia and cardiovascular disease on frailty status of community-dwelling older women: the Women's Health and Aging Studies I and II. J Gerontol A Biol Sci Med Sci. juin 2005;60(6):729-35.

19. Malmstrom TK, Miller DK, Morley JE. A comparison of four frailty models. J Am Geriatr Soc. avr 2014;62(4):721-6.

20. Amalou L, Roca F, François F, Druesne L, Langlois R, Pesqué T, et al. Tracking tool for frailty: ABCDEF. Geriatr Psychol Neuropsychiatr Vieil. 1 sept 2016;14(3):287-95.

21. Dent E, Kowal P, Hoogendijk EO. Frailty measurement in research and clinical practice: A review. Eur J Intern Med. juin 2016;31:3-10.

22. Subra J, Gillette-Guyonnet S, Cesari M, Oustric S, Vellas B, Platform Team. The integration of frailty into clinical practice: preliminary results from the Gérontopôle. J Nutr Health Aging. août 2012;16(8):714-20.

23. Rapport d'évaluation HAS. Nouveaux modes d'organisation des soins destinés à optimiser les parcours de soins des personnes âgées en risque de perte d'autonomie. Haute Autorité de santé; 2015.

24. Recommandation HAS. Comment repérer la fragilité en soins ambulatoires ? Haute Autorité de santé; $\quad 1^{\text {er }} \quad$ juin $2013 . \quad$ https://webzine.hassante.fr/portail/upload/docs/application/pdf/2013-06/fiche_parcours_fragilite_vf.pdf

25. Abellan van Kan G, Rolland Y, Andrieu S, Bauer J, Beauchet O, Bonnefoy M, et al. Gait speed at usual pace as a predictor of adverse outcomes in community-dwelling older people an International Academy on Nutrition and Aging (IANA) Task Force. J Nutr Health Aging. déc 2009;13(10):881 9.

26. Chainani V, Shaharyar S, Dave K, Choksi V, Ravindranathan S, Hanno R, et al. Objective measures of the frailty syndrome (hand grip strength and gait speed) and cardiovascular mortality: A systematic review. Int J Cardiol. 15 juill 2016;215:487-93.

27. López-Teros T, Gutiérrez-Robledo LM, Pérez-Zepeda MU. Gait Speed and Handgrip Strength as Predictors of Incident Disability in Mexican Older Adults. J Frailty Aging. 2014;3(2):109-12. 
28. Åhlund K, Bäck M, Öberg B, Ekerstad N. Effects of comprehensive geriatric assessment on physical fitness in an acute medical setting for frail elderly patients. Clin Interv Aging. 2017;12:1929-39.

29. Pao Y-C, Chen C-Y, Chang C-I, Chen C-Y, Tsai J-S. Self-reported exhaustion, physical activity, and grip strength predict frailty transitions in older outpatients with chronic diseases. Medicine (Baltimore). juin 2018;97(23):e10933.

30. Studenski S, Perera S, Patel K, Rosano C, Faulkner K, Inzitari M, et al. Gait speed and survival in older adults. JAMA. 5 janv 2011;305(1):50-8.

31. Lundin H, Sääf M, Strender L-E, Nyren S, Johansson S-E, Salminen H. Gait speed and oneleg standing time each add to the predictive ability of FRAX. Osteoporos Int. 2017;28(1):179 $-87$.

32. Alley DE, Hicks GE, Shardell M, Hawkes W, Miller R, Craik RL, et al. Meaningful improvement in gait speed in hip fracture recovery. J Am Geriatr Soc. sept 2011;59(9):1650 $-7$

33. Lee L, Patel T, Costa A, Bryce E, Hillier LM, Slonim K, et al. Screening for frailty in primary care: Accuracy of gait speed and hand-grip strength. Can Fam Physician. janv 2017;63(1):e51-7.

34. Zuckerman J, Ades M, Mullie L, Trnkus A, Morin J-F, Langlois Y, et al. Psoas Muscle Area and Length of Stay in Older Adults Undergoing Cardiac Operations. Ann Thorac Surg. mai 2017;103(5):1498-504.

35. Cruz-Jentoft AJ, Baeyens JP, Bauer JM, Boirie Y, Cederholm T, Landi F, et al. Sarcopenia: European consensus on definition and diagnosis: Report of the European Working Group on Sarcopenia in Older People. Age Ageing. juill 2010;39(4):412-23.

36. Landi F, Cruz-Jentoft AJ, Liperoti R, Russo A, Giovannini S, Tosato M, et al. Sarcopenia and mortality risk in frail older persons aged 80 years and older: results from ilSIRENTE study. Age Ageing. mars 2013;42(2):203-9.

37. Castell MV, van der Pas S, Otero A, Siviero P, Dennison E, Denkinger M, et al. Osteoarthritis and frailty in elderly individuals across six European countries: results from the European Project on OSteoArthritis (EPOSA). BMC Musculoskelet Disord. 17 nov 2015;16:359.

38. Li B-L, Li W, Bi J-Q, Meng Q-G, Fei J-F. Predicting factors associated with frailty in aged patients with bone-arthrosis pain in the clinic. Phys Sportsmed. 2016;44(4):391-6.

39. Schuurmans H, Steverink N, Lindenberg S, Frieswijk N, Slaets JPJ. Old or frail: what tells us more? J Gerontol A Biol Sci Med Sci. sept 2004;59(9):M962-965.

40. Johnson RL, Abdel MP, Frank RD, Chamberlain AM, Habermann EB, Mantilla CB. Impact of Frailty on Outcomes After Primary and Revision Total Hip Arthroplasty. J Arthroplasty. janv 2019;34(1):56-64.e5. 
41. Nawrot J, Boonen A, Peeters R, Starmans M, van Onna M. Rheumatologists' Views and Experiences in Managing Rheumatoid Arthritis in Elderly Patients: A Qualitative Study. J Rheumatol. mai 2018;45(5):590-4.

42. Andrews JS, Trupin L, Yelin EH, Hough CL, Covinsky KE, Katz PP. Frailty and reduced physical function go hand in hand in adults with rheumatoid arthritis: a US observational cohort study. Clin Rheumatol. mai 2017;36(5):1031-9.

43. Ensrud KE, Ewing SK, Taylor BC, Fink HA, Stone KL, Cauley JA, et al. Frailty and risk of falls, fracture, and mortality in older women: the study of osteoporotic fractures. J Gerontol A Biol Sci Med Sci. juill 2007;62(7):744-51.

44. Kim HJ, Park S, Park SH, Park J, Chang BS, Lee CK, et al. Prevalence of Frailty in Patients with Osteoporotic Vertebral Compression Fracture and Its Association with Numbers of Fractures. Yonsei Med J. mars 2018;59(2):317-24.

45. Gärdsell P, Johnell O, Nilsson BE, Nilsson JA. The predictive value of fracture, disease, and falling tendency for fragility fractures in women. Calcif Tissue Int. déc 1989;45(6):327-30.

46. Vellas BJ, Wayne SJ, Garry PJ, Baumgartner RN. A two-year longitudinal study of falls in 482 community-dwelling elderly adults. J Gerontol A Biol Sci Med Sci. juill 1998;53(4):M264-274.

47. Podsiadlo D, Richardson S. The timed «Up \& Go »: a test of basic functional mobility for frail elderly persons. J Am Geriatr Soc. févr 1991;39(2):142-8.

48. Guralnik JM, Simonsick EM, Ferrucci L, Glynn RJ, Berkman LF, Blazer DG, et al. A short physical performance battery assessing lower extremity function: association with selfreported disability and prediction of mortality and nursing home admission. J Gerontol. mars 1994;49(2):M85-94.

49. Briot K, Roux C, Thomas T, Blain H, Buchon D, Chapurlat R, et al. 2018 update of French recommendations on the management of postmenopausal osteoporosis. Joint Bone Spine. oct 2018;85(5):519-30.

50. Rolland Y, Abellan Van Kan G, Gillette-Guyonnet S, Roux C, Boonen S, Vellas B. Strontium ranelate and risk of vertebral fractures in frail osteoporotic women. Bone. févr 2011;48(2):332-8.

51. Souberbielle J-C, Benhamou CL, Cortet B, Rousière M, Roux C, Abitbol V, et al. Rapport de la HAS sur les dosages de vitamine $\mathrm{D}$ : ne passons pas d'une situation extrême à une autre situation tout aussi extrême. La Presse Médicale. janv 2014;43(1):5-8.

52. Pilz S, Dobnig H, Tomaschitz A, Kienreich K, Meinitzer A, Friedl C, et al. Low 25hydroxyvitamin $\mathrm{D}$ is associated with increased mortality in female nursing home residents. J Clin Endocrinol Metab. avr 2012;97(4):E653-657.

53. Deo R, Katz R, Shlipak MG, Sotoodehnia N, Psaty BM, Sarnak MJ, et al. Vitamin D, parathyroid hormone, and sudden cardiac death: results from the Cardiovascular Health Study. Hypertension. déc 2011;58(6):1021-8. 
54. Semba RD, Houston DK, Ferrucci L, Cappola AR, Sun K, Guralnik JM, et al. Low serum 25-hydroxyvitamin D concentrations are associated with greater all-cause mortality in older community-dwelling women. Nutr Res. août 2009;29(8):525-30.

55. Semba RD, Houston DK, Bandinelli S, Sun K, Cherubini A, Cappola AR, et al. Relationship of 25-hydroxyvitamin $\mathrm{D}$ with all-cause and cardiovascular disease mortality in older community-dwelling adults. Eur J Clin Nutr. févr 2010;64(2):203-9.

56. Smit E, Crespo CJ, Michael Y, Ramirez-Marrero FA, Brodowicz GR, Bartlett S, et al. The effect of vitamin D and frailty on mortality among non-institutionalized US older adults. Eur J Clin Nutr. sept 2012;66(9):1024-8.

57. Shardell M, D’Adamo C, Alley DE, Miller RR, Hicks GE, Milaneschi Y, et al. Serum 25hydroxyvitamin $\mathrm{D}$, transitions between frailty states, and mortality in older adults: the Invecchiare in Chianti Study. J Am Geriatr Soc. févr 2012;60(2):256-64.

58. Keshishian A, Boytsov N, Burge R, Krohn K, Lombard L, Zhang X, et al. Examining the Effect of Medication Adherence on Risk of Subsequent Fracture Among Women with a Fragility Fracture in the U.S. Medicare Population. J Manag Care Spec Pharm. nov 2017;23(11):1178-90. 


\section{FIGURES ET TABLEAUX}

Figure 1 : Cycle de la Fragilité selon la conception phénotypique de Fried d'après Fried et col. [1].

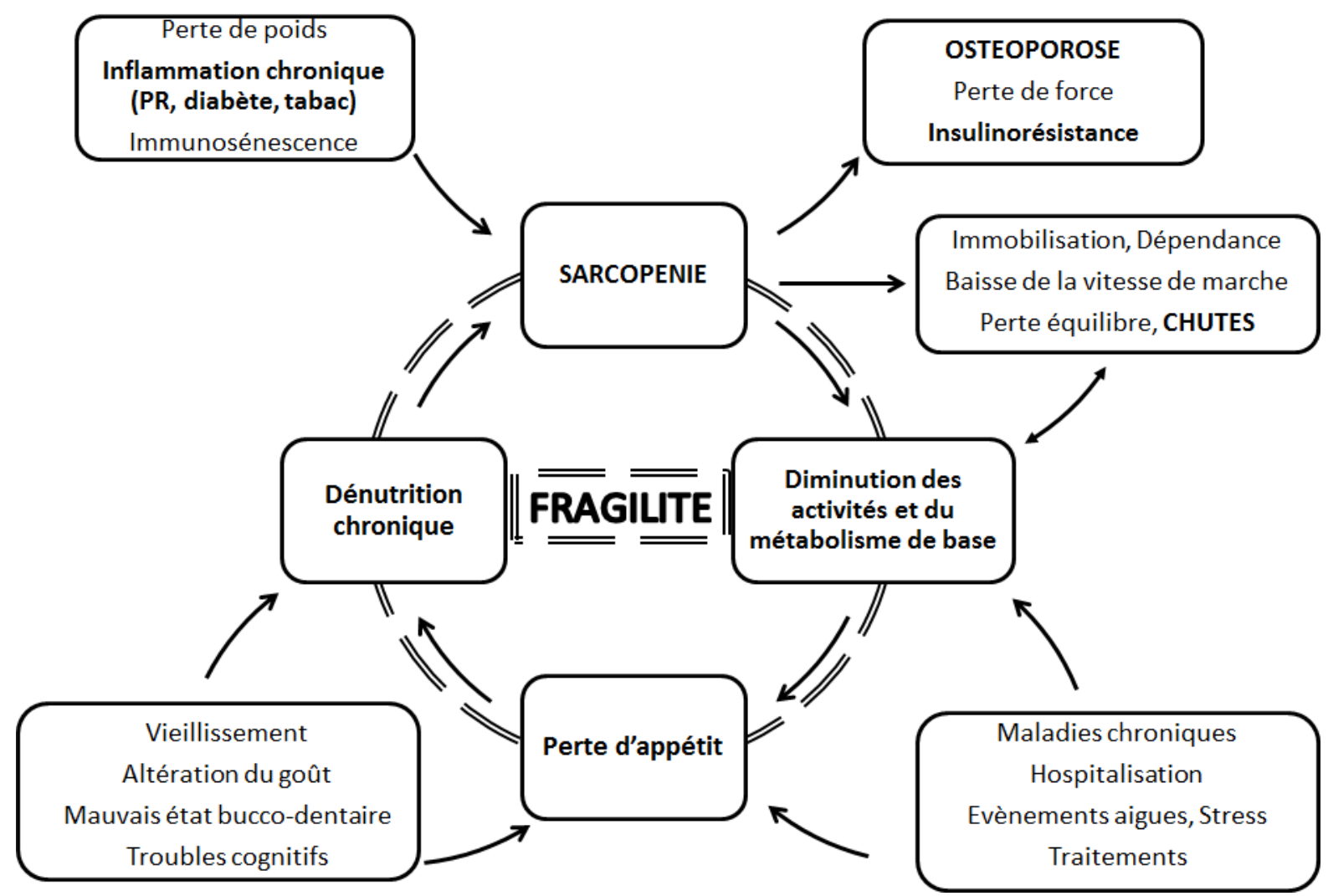


Figure 2: Positionnement du phénotype de la fragilité et de ses conséquences :

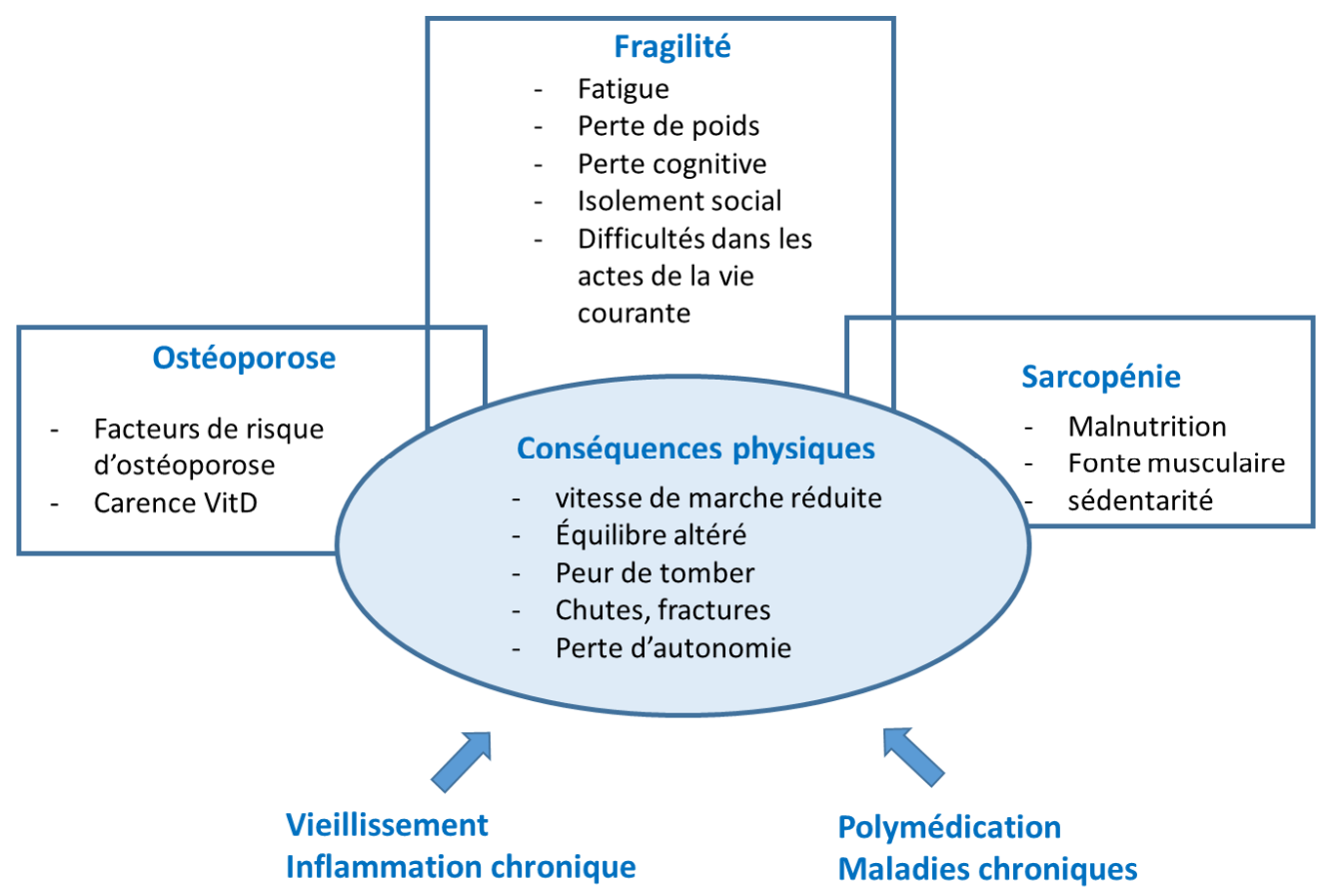


Figure 3 : Modalités de réalisation de la mesure de la vitesse de marche sur 4 mètres d'après Abellan et col. [25]



- Le test de marche sur 4 mètres est réalisé sur une distance minimale de huit mètres en incluant les deux mètres d'accélération et de décélération.

- La vitesse de marche ne peut être mesurée que si la personne a acquis un rythme de marche sur quelques mètres après l'initiation du mouvement.

- Nécessité de déterminer un couloir dédié au test de marche d'une longueur de 10 mètres avec:

- un point fixe marqué au sol pour commencer à chronométrer le départ

- des instructions au patient « marchez à votre rythme habituel, à votre aise » et associant aux consignes les encouragements et l'annonce du processus de chronométrage 
Figure 4 : Exemple de dynamomètre à main

La mesure de force musculaire par dynamomètre permet de déterminer la force de préhension. Il faut effectuer 3 mesures de la main dominante préférentiellement et calculer la moyenne des 3 mesures consécutives, chez un patient assis ou debout confortablement (coude fléchi à $90^{\circ}$, l'avant-bras et le poignet dans une position neutre). Le soignant demande au patient de serrer la poignée du dynamomètre avec la force maximum (Photo CHR d'Orléans - Court Séjour Gériatrie)

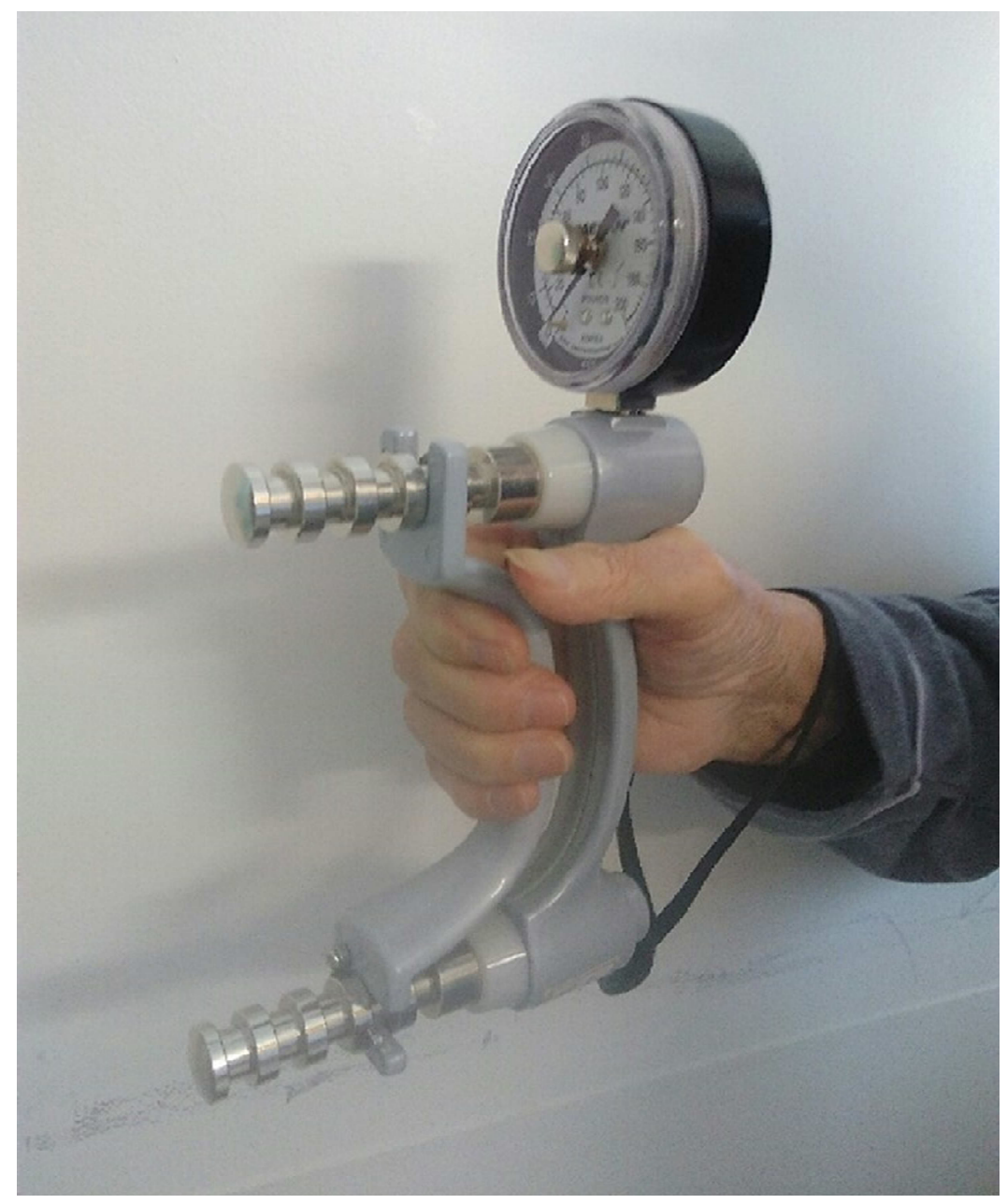


Figure 5 : Gérontopôle Frailty Screening Tool (GSFT) : patients de 65 ans et plus, autonomes $(\mathrm{ADL} \geq 5 / 6)$, à distance de toute pathologie aiguë [22, 24].

Patients de 65 ans et plus, autonomes (ADL 6/6), à distance de toute pathologie aiguë

\section{DÉPIST AGE}

OUI NON NE SAIT PAS

Votre patient vit-il seul?

Votre patient a-t-il perdu du poids ces 3 derniers mois?

Votre patient s'est-il senti plus fatigué ces 3 derniers mois ?

Votre patient a-t-il plus de difficultés pour se déplacer depuis ces 3 derniers mois?

Votre patient se plaint-il de problèmes de mémoire?

Votre patient présente-t-il une vitesse de marche ralentie (plus de 4 secondes pour parcourir 4 mètres) ?

Si vous avez répondu OUI à l'une de ces questions :

Votre patient vous paraît-il $\square$ OUI $\sqsupset \mathrm{NON}$ fragile?

Si OUI, votre patient accepte-t-il de participer à une évaluation de sa fragilité en hôpital de jour ?
$\square$ OUI
$\square \mathrm{NON}$ 
Tableau 1: Caractéristiques de la fragilité et outils de mesure selon le concept phénotypique de Fried [1,9]

\begin{tabular}{ll}
\hline Phénotypes & Critères de mesures \\
\hline Amaigrissement & Perte de poids $>4.5 \mathrm{~kg}$ ou $\geq 5 \%$ par an \\
Faiblesse/fatigue/symptômes & Sensation d'épuisement 3-4 jours par semaine ou plus \\
dépressifs & selon l'échelle d'autoévaluation US Center for \\
& Epidemiological Studies depression scale [9] \\
Baisse des activités & Dépenses énergétiques : \\
physiques/sédentarité & $<383 \mathrm{Kcal} / \mathrm{semaine}$ (Homme) \\
& $<270 \mathrm{Kcal} / \mathrm{semaine}($ Femme) \\
Vitesse de marche lente & Vitesse de marche $<0.8 \mathrm{~m} / \mathrm{seconde}$ \\
Diminution de la force de & Force de préhension : Grip strenght test modulé selon \\
préhension & la taille et l'indice de masse corporelle \\
\hline
\end{tabular}


Tableau 2 : Démarche anthropométrique pour calculer la Surface Musculaire Brachiale (SMB)

\begin{tabular}{|l|l|}
\hline Démarche: & Mesures et calculs nécessaires \\
\hline Mesurer & $\begin{array}{l}* \text { La circonférence brachiale }(\mathrm{CB}, \mathrm{cm}) \\
* \text { le pli cutané tricipital }(\mathrm{PCT}, \mathrm{cm})\end{array}$ \\
\hline circonférence musculaire brachiale $(\mathrm{CMB})$ & $\mathrm{CMB}=\mathrm{CB}-[\pi \times \mathrm{PCT}]$ \\
\hline surface musculaire brachiale $\left(\mathrm{cm}^{2}\right)$ & $\mathrm{SMB}=\mathrm{CMB}^{2} / 4 \pi$ \\
\hline
\end{tabular}







Appui unipodal $(\mathbf{t}<5 \mathrm{sec})[46]$

Expliquer:

-La nécessité de rester le plus longtemps possible en appui unipodal sur la jambe de son choix.

-La jambe relevée ne doit pas toucher l'autre et arriver au moins à mimollet.

-Sur les «3 mesures consécutives réalmisées, la meilleure performance est conservée.

\section{Le maintien en équilibre sur une jambe moins de 5 sec est anormal.}

Timed Up and Go test [47]

-Se lever d'une chaise sans utiliser les accoudoirs,

-Marcher sur 3 mètres, faire demi-tour et retourner s'assoir.

Anomalie au-dessus de 20 sec.

Pieds-tandem [48]

Pieds joints / semi-tandem / tandem (équilibre étudié $10 \mathrm{sec}$ sur chaque position)

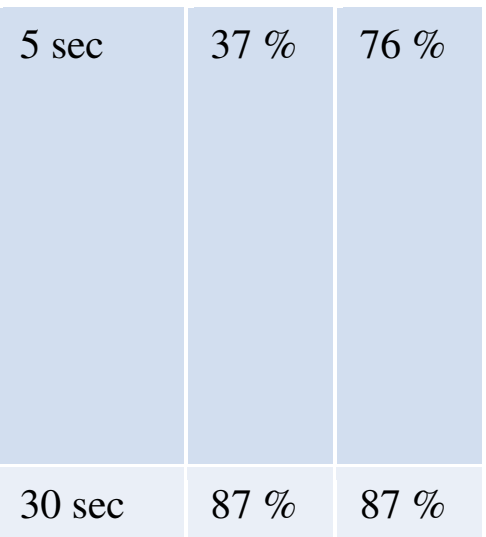

$30 \mathrm{sec}$

Les tests standardisés ci-dessus doivent être interprétés avec prudence en fonction de la pathologie rhumatologique en cours de traitement et des autres comorbidités pouvant interférer sur les potentialités de la marche. Au-delà du score enregistré, l'interprétation qualitative de la stabilité, du balancement des bras à la marche, d'une peur à la verticalisation, de la réactivité face aux obstacles (réactions parachutes) et des stratégies compensatrices mises en place par la personne âgée sont à évaluer conjointement et à consigner pour suivre l'évolution des performances et adapter les recommandations éventuelles. 